\title{
Keratitis from abuse of corneal anaesthetics
}

\author{
HAROLD E. HENKES AND THEODOR N. WAUBKE \\ From the University Eye Clinics, Rotterdam and Essen
}

SUMMARY Topically applied anaesthetics are potentially dangerous, as frequent and continuous application may lead to anaesthetic-induced keratitis. Three patients with serious corneal lesions are described.

Physicians sometimes fail to realise that topical application of anaesthetics to the eye may lead to severe corneal lesions and permanent reduction of visual acuity. Such a reaction can be evoked in any eye that has been subjected to prolonged application of topical anaesthetics as a means of relieving the discomfort of minor injuries. Although the topical use of these drugs is widespread, relatively few cases have been published of misuse of corneal anaesthetics. This may be due to the fact that damage done to the cornea is mostly limited, and full vision is usually restored.

However, chronically applied corneal anaesthetics cause delay in corneal epithelial healing and produce corneal oedema and epithelial erosions, leading to increased pain. This leads to shortened intervals between applications. Loss of the epithelial barrier may then set the stage for infection and permanent corneal scarring.

Of the 18 cases reported between 1968 and 1973 (Epstein and Paton, 1968; Willis and Laibson, 1970; Hilsdorf and Zenklusen, 1973) 5 were medical professionals. They had treated themselves for a few days up to 2 weeks. It would appear that an occupational hazard exists, since a quarter of all the cases were doctors. Five more cases (no doctors) were seen in the Rotterdam Eye Hospital and in the University Eye Clinic at Essen, Germany. Three cases in which serious loss of vision occurred will be described.

\section{Case reports}

CASE 1

A 40-year-old man was hit by a chemical fluid in his right eye. The eye was slightly irritated and the cornea showed an erosion. Oxybuprocaine

Address for reprints: Professor H. E. Henkes, Oogziekenhuis, Schiedamsevest 180, Rotterdam 3001, Holland
(Novesine) was prescribed by the ophthalmologist for pain relief. The patient continued to use the drug owing to persisting pain, applying it as often as every 30 minutes for 6 weeks.

When he was referred for examination the visual acuity had dropped to hand movements. Examination revealed an allergic reaction of the skin and the eyelids. The conjunctiva was markedly injected. Half the epithelium was missing from the central cornea, and under the eroded epithelium there was full-thickness corneal oedema. Sensibility of the cornea was lost. Superficial and stromal vascularisation existed, and in addition to the anaestheticinduced keratitis signs of anterior uveitis were clearly present (Fig. 1).

The patient was admitted to hospital. Oxybuprocaine drops were discontinued, and the patient was started on a course of firm pressure patching and cycloplegia with atropine $1 \%$. Strong analgesics had to be given for intolerable pain during the next few days. Five days later the pain was con-

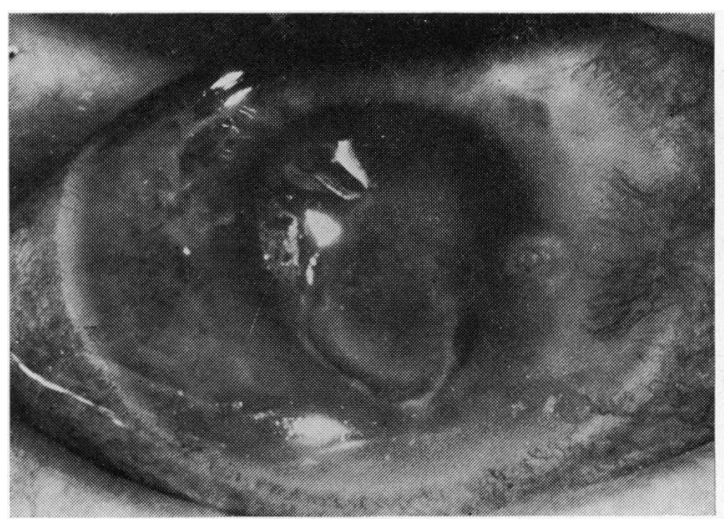

Fig. 1 Case 1. Corneal ulceration in a case of anaesthetic-induced keratitis following a 6 weeks' application of oxybuprocaine drops 
siderably relieved, and on removing the patch it was noted that epithelisation had started and stromal oedema had diminished.

The cornea partly cleared in about 3 weeks, but stromal infiltrate was still present. In the next 6 weeks stromal oedema was further reduced, but it became clear that a thick leucoma in the superficial stroma would persist in the central cornea.

Four months after oxybuprocaine medication was discontinued the leucoma was vascularised, and vision in the right eye was finger-counting at $1 \mathrm{~m}$ (Fig. 2). Keratoplasty was advised but rejected by the patient. Three years later the patient was examined again. The condition had not changed essentially.

\section{CASE 2}

A 32-year-old man suffered a painful erosion of the right eye. His ophthalmologist supplied him with a bottle of oxybuprocaine (Chibro-Kerakain), to be returned the next day. The patient, however, continued the medication as the pain returned in the period between the instillations. Notwithstanding an increased frequency of application, the periods of relief of pain became shorter and shorter. As the pain became intractable the patient visited the outpatient department 2 weeks later.

On examination the cornea of the right eye showed a centrally located erosion, with full-thickness stromal infiltration and keratitic precipitates. Vision had decreased to finger-counting at $3 \mathrm{~m}$.

He was admitted to hospital and treatment was started with, among other things, mydriatics and antibiotics. The patient avoided mentioning the continuous use of oxybuprocaine drops, because he was afraid to lose a medication on which he felt entirely dependent. Thus he managed to continue for several weeks the instillation of oxybuprocaine

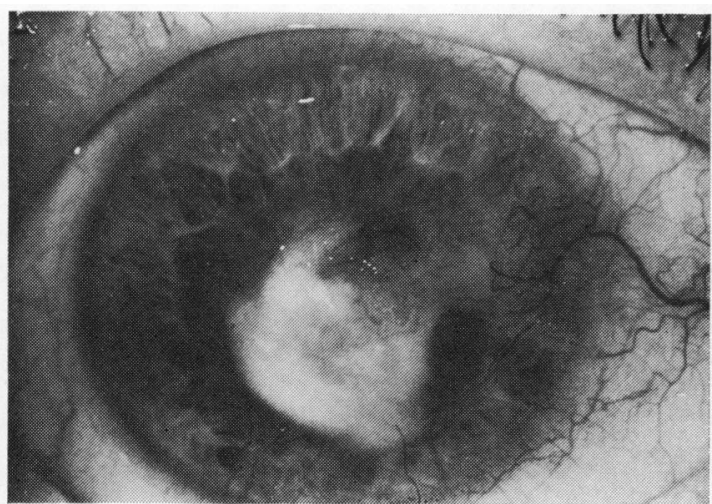

Fig. 2 Case 1. Four months after discontinuation of anaesthetic drops. A corneal leucoma has persisted

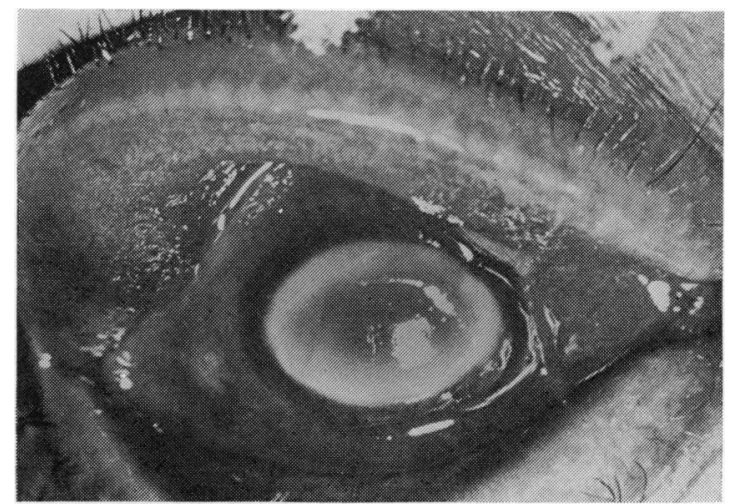

Fig. 3 Case 2. Serious oxybuprocaine-induced keratitis, showing additional allergic oedema of conjunctiva

drops, replacing at night the empty bottles by filled ones. Despite thorough treatment the corneal ulceration progressed steadily (Fig. 3). Corneal sensitivity was completely lost. The possibility of self-medication was suggested more than once, but this idea was emphatically denied. A thorough search of the room for incriminating material was unsuccessful. The situation seemed desperate until the patient was found asleep with a bottle of oxybuprocaine in his hand.

CASE 3

At about the same time as the previous case, a man of 32 years presented himself at the outpatient department complaining of severe pain in the right eye due to an erosion of the cornea. Three days before he had been struck in the eye. He went to the ophthalmologist (by chance the same one who treated case 2) and received a bottle of oxybuprocaine (Chibro-Kerakain) to relieve the pain, to be returned the next day. But he too continued to apply the anaesthetic drops owing to recurring pain.

The patient was treated in the outpatient department with mydriatics and antibiotics, but his condition deteriorated. An allergic reaction of the eyelids surrounding skin, and fingertips developed (Figs. 4 and 5). As a progressive ulcer of the cornea developed and the patient complained of intolerable pain (though sensibility of the cornea was almost totally lost), he was admitted to hospital.

Despite intensive treatment with antiallergic and antibiotic drugs the condition deteriorated. The patient started to complain of his sound eye, and a central erosion of the cornea was found in it. At about the same time patient No. 2 also developed an erosion of the fellow eye, with photophobia and severe pain. Owing to the similarity of the unusual resistance to treatment of the 2 patients they 


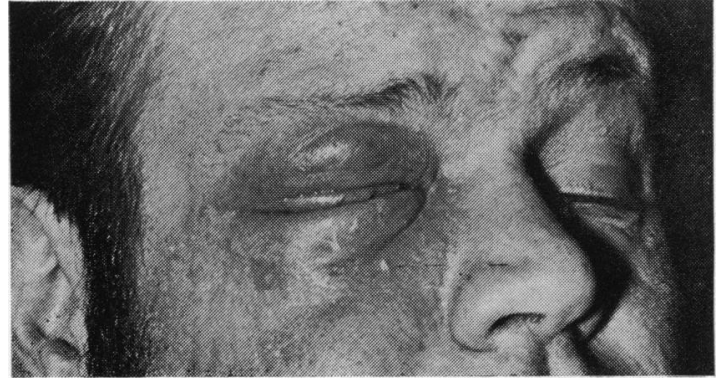

Fig. 4 Case 3. Allergic eczema of eyelids due to use of oxybuprocaine

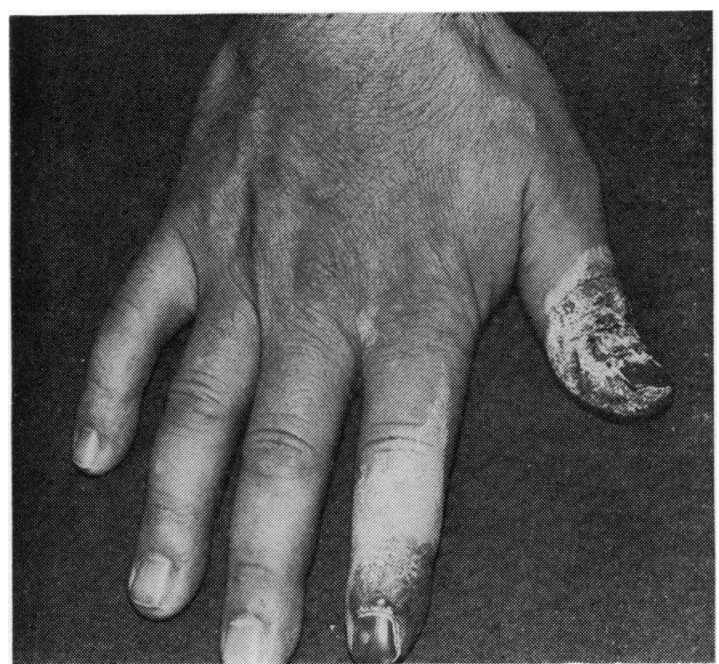

Fig. 5 Case 3. Allergic eczema of fingers were accommodated in the same hospital room.

Soon they both found out that neither of them could do without the anaesthetic, and so they secretly obtained fresh bottles of oxybuprocaine.

After the bottle of anaesthetic was discovered in the hand of patient No. 2, the condition of the eyes of both patients improved. In both, however, a pronounced vascularised leucoma resulted. Perforating keratoplasty had to be performed, with a favourable outcome in patient No. 2 (Fig. 6). Visual acuity improved to $0 \cdot 3$. In the third patient, however, even renewed perforating keratoplasty did not lead to a favourable outcome.

\section{Discussion}

Soon after its surface anaesthetic property was recognised cocaine applied to the cornea was found to cause epithelial erosion. Newer synthetic topical anaesthetics have potentially the same serious implications, as these drugs are derivatives of cocaine. They include tetracaine, butacaine, proparacaine, and oxybuprocaine. The clinical findings in anaesthetic-induced keratitis are similar to those observed in neuroparalytic keratitis. The resulting corneal anaesthesia leads to decreased reflex blinking and consequently increased susceptibility to drying, foreign bodies, trauma, and infection. Topical anaesthetics, moreover, inhibit corneal respiration and glucose metabolism, increase corneal permeability, and decrease mitotic epithelial activity. It is possible that locally applied anaesthetics interfere with epithelial metabolism by impairing the trophic function of the corneal nerve fibres. This too may

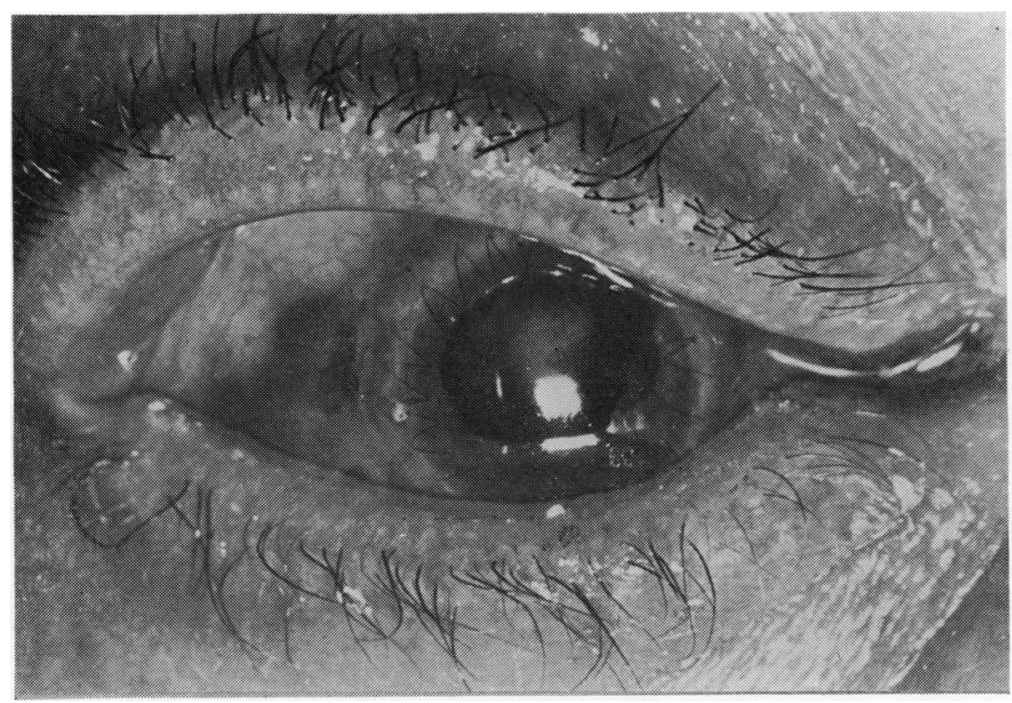

Fig. 6 Result of perforating keratoplasty in case 2. Vision improved to 0.3 
result in intolerable pain of the insensitive cornea, anaesthesia dolorosa, which may force the patient to apply the anaesthetic drops more and more frequently. This may make the withdrawal of the anaesthetic in some patients impossible without administering massive doses of strong analgesics.

Clearly physicians should avoid the use and prescription of such agents except as office and clinic procedures. 'The topical anaesthetic is a good friend but may become an enemy when used too often.' (Willis and Laibson, 1970.)

\section{References}

Epstein, D. L., and Paton, D. (1968). New England Journal of Medicine, 279, 396.

Hilsdorf, C., and Zenklusen, G. (1973). Klinische Monatsblätter für Augenheilkunde, 162, 525.

Willis, W. E., and Laibson, P. R. (1970). Canadian Journal of Ophthalmology, 5, 239. 